\title{
Evaluation of DoP-CPD Classification Technique and Multi Looking Effects for RADARSAT-2 Images
}

\author{
Kyung-Yup Lee ${ }^{\star \dagger}$, Yisok Oh** and Younsoo Kim* \\ * Satellite Data Application Department, Korea Aerospace Research Institute \\ ** School of Electronic and Electrical Engineering, Hongik University
}

\begin{abstract}
This paper give further assessment on the original DoP-CPD classification scheme. This paper provides some additional comparative study on the DoP-CPD with H/A/alpha classifier in terms of multi look effects and classification performances. The statistics and multi looking effects of the DoP and $\mathrm{CPD}$ were analyzed with measured polarimetric SAR data. DoP-CPD is less sensitive to the number of averaging pixels than the entropy-alpha technique. A DoP-CPD diagram with appropriate boundaries between six different classes was then developed based on the data analysis. A polarimetric SAR image DoP-CPD classification technique is verified with C-band polarimetric RADARSAT-2 images.
\end{abstract}

Key Words : SAR, Classification, Degree of polarization, Co-polarized phase-difference, Radasat-2

\section{Introduction}

There are various supervised and unsupervised classification algorithms for polarimetric SAR(Kong et al., 1988; Lee et al., 1994; Lardeux et al., 2009; Ersahin et al., 2010). There are also various PolSAR decomposition techniques (Van zyl et al., 1987; Freeman and Durden, 1998; Yamaguchi et al., 2005; Lee and Oh, 2009). The entropy-alpha-anisotropy technique is the most common classification technique for polarimetric SAR images (Cloude and Pottier, 1997). The Cloude-Pottier technique is to classify the polarimetric response of each pixel into an entropy-alpha-anisotropy diagram, in which the entropy, alpha and anisotropy are computed from
Eigen analysis of the coherency matrices. Although the Eigen analysis may be an excellent technique to analyze an information matrix, it has some weak points for the SAR image classification. In the process of the Eigen analysis, the magnitudes of the coherency matrix elements are normalized such that the information in the magnitudes is lost, and consequently it prevents us to proceed to the classification process after the Eigen analysis. So, in our previous research, we proposed one unsupervised classification technique using the degree of polarization (DoP) and the co-polarized phasedifference (CPD) statistics (Oh et al., 2009; Rio et al., 2006; Ulaby et al., 1992). This classification technique is simple and intuitive because the DoP and

\footnotetext{
Received April 3, 2012; Revised May 4, 2012, Revised May 20, 2012; Accepted May 22, 2012.

${ }^{\dagger}$ Corresponding Author: Kyung-Yup Lee (ky_lee@kari.re.kr)
} 
CPD are physical parameters which can be obtained directly from the covariance matrix, or the Stokes scattering matrix, without Eigen analysis. We proposed a DoP-CPD technique for classification boundary values from experimental data using only L-band polarimetric AirSAR and PALSAR data. Hence, in this paper DoP-CPD classification technique is verified with $\mathrm{C}$-band polarimetric RADARSAT-2 image. In addition, the multi looking effects on the DoP and CPD are also analyzed. Speckle complicates the image interpretation problem and reduces the accuracy of image segmentation and classification. We analyzed the sensitivities of the classification parameters on various targets and the effect of ensemble averaging. The DoP-CPD diagram boundary values are dependent on the number of averaging pixels.

\section{Classification parameters}

In general, the scattered electric field $\mathrm{E}^{s}$ can be written with the complex $2 \times 2$ scattering matrix $S$ (Sinclair, 1950):

$$
\left[\begin{array}{c}
E_{h}^{s} \\
E_{v}^{s}
\end{array}\right]=\frac{e^{-j k_{0} r}}{r}\left[\begin{array}{l}
S_{h h} S_{h v} \\
S_{v h} S_{v v}
\end{array}\right]\left[\begin{array}{c}
E_{h}^{i} \\
E_{v}^{i}
\end{array}\right]
$$

where $h$ and $v$ denote horizontally and vertically polarized waves, respectively. The Stokes vector is defined as

$$
\bar{F}^{s}=\left(\begin{array}{c}
I^{s} \\
Q^{s} \\
U^{s} \\
V^{s}
\end{array}\right)==\left(\begin{array}{c}
<\left|E_{h}^{s} E_{h}^{s *}\right|>+<\left|E_{v}^{s} E_{v}^{s *}\right|> \\
<\left|E_{h}^{s} E_{h}^{s *}\right|>-<\left|E_{v}^{s} E_{v}^{s *}\right|> \\
<2 \operatorname{Re}\left(E_{h}^{s} E_{v}^{s *}\right)> \\
<2 \operatorname{Im}\left(E_{h}^{s} E_{v}^{s *}\right)>
\end{array}\right)
$$

where $\langle\cdots\rangle$ indicates the ensemble average (Ulaby and Elachi, 1990). The DoP of the partially polarized wave is then defined as

$$
D o P=\frac{\sqrt{Q^{s 2}+U^{s 2}+V^{s 2}}}{I^{s}}
$$

The DoP can be obtained from the Mueller matrix, the Stokes scattering matrix, or the covariance matrix for $\mathrm{h}$ - and v-polarized wave incidences. We used then the DoP value which is the mean of the $\mathrm{h}$ - and vpolarized DoP values.

The DoP could be used as an important factor for classifying image classes, because the DoP measures how much the effect of multiple reflections is contained in a target area (Elies et al., 1997).

The CPD can be defined as the ensemble average of the co-polarized phase-difference between the $h h$ and $v v$-polarized scattered waves. In the backscattering case, the measured scattering matrix by a polarimetric radar system consists of five quantities; $\left|\mathrm{S}_{\mathrm{hh}}\right|,\left|\mathrm{S}_{\mathrm{vv}}\right|$ and $\mathrm{IS} \mathrm{hv} \mid, \Phi_{\mathrm{c}}=\Phi_{\mathrm{hh}}-\Phi_{\mathrm{vv}}$, and $\Phi_{\mathrm{x}}=\Phi_{\mathrm{hv}}-\Phi_{\mathrm{vv}}$.

$$
\mathrm{S}=e^{-i \phi_{v v}}\left[\begin{array}{cc}
\left|s_{h h}\right| e^{-i \phi_{c}} & \left|s_{h v}\right| e^{-i \phi_{x}} \\
\left|s_{v h}\right| e^{-i \phi_{x}} & \left|s_{v v}\right|
\end{array}\right]
$$

The $\operatorname{CPD} \Phi_{c}$ can be written as

$$
\phi_{c}=\phi_{h h}-\phi_{v v}=\tan ^{-1}\left(\frac{<\operatorname{Im}\left(S_{h h} S_{v v}^{*}\right)>}{\left.<\operatorname{Re}\left(S_{h h} S_{v v}^{*}\right)\right\rangle}\right)
$$

For most natural targets, the cross-polarized phase difference $\Phi_{\mathrm{x}}$ is almost uniformly distributed over [0, $2 \pi$ ], and therefore, contains less target-specific information. On the other hand, the $\operatorname{CPD}\left(\Phi_{\mathrm{c}}\right)$ shows a Gaussian PDF with both a standard deviation and a mean which depend on the target characteristics. Hence, the CPD contains some target-specific information (Ulaby et al., 1992). For extreme cases, for example, $\mathrm{CPD}=0^{\circ}$ for radar scattering from a flat surface, and $\mathrm{CPD}=180^{\circ}$ for a dihedral-type scattering. Otherwise, the CPD has various values depending on the combination of various scattering mechanisms: single-bounce scattering, double bounce scattering, and volume scattering. Hence, the feature of $\Phi_{c}$ can be a good parameter for classifying polarimetric SAR data. 


\section{Effects of multi looking on the DoP and CPD}

Speckle in SAR images is a scattering phenomenon. Speckle complicates the image interpretation problem and reduces the accuracy of image segmentation and classification. Speckle

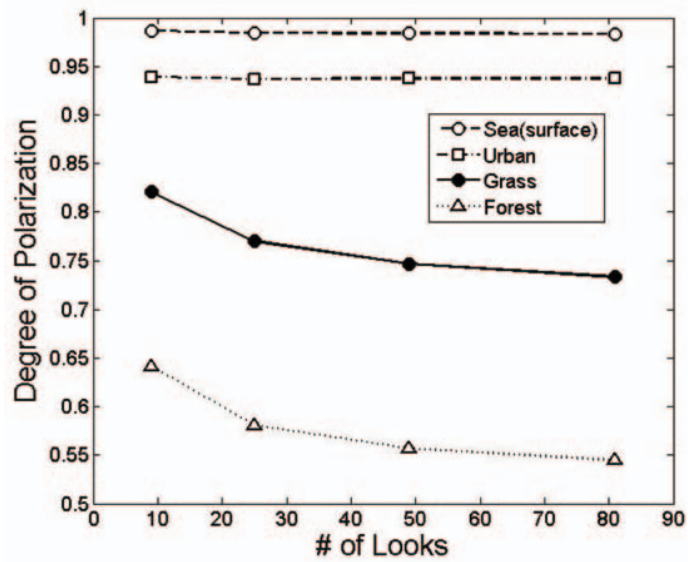

Fig. 1. Behaviors of the DoP with various numbers of looks for sea, urban, short vegetation and tall vegetation. filtering and multilook processing is a procedure commonly adopted to reduce the noise effect. The most commonly applied technique is the boxcar filter, which replaces the center pixel in a moving window size $3 \times 3$ or larger with average of pixels in the window. The effects of ensemble averages on the DoP and CPD are examined by increasing the

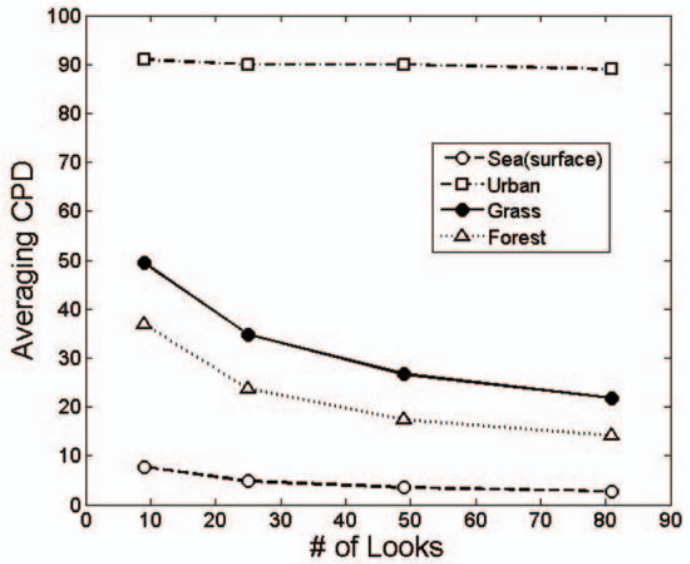

Fig. 2. Behaviors of the CPD with various numbers of looks for sea, urban, short vegetation and tall vegetation.
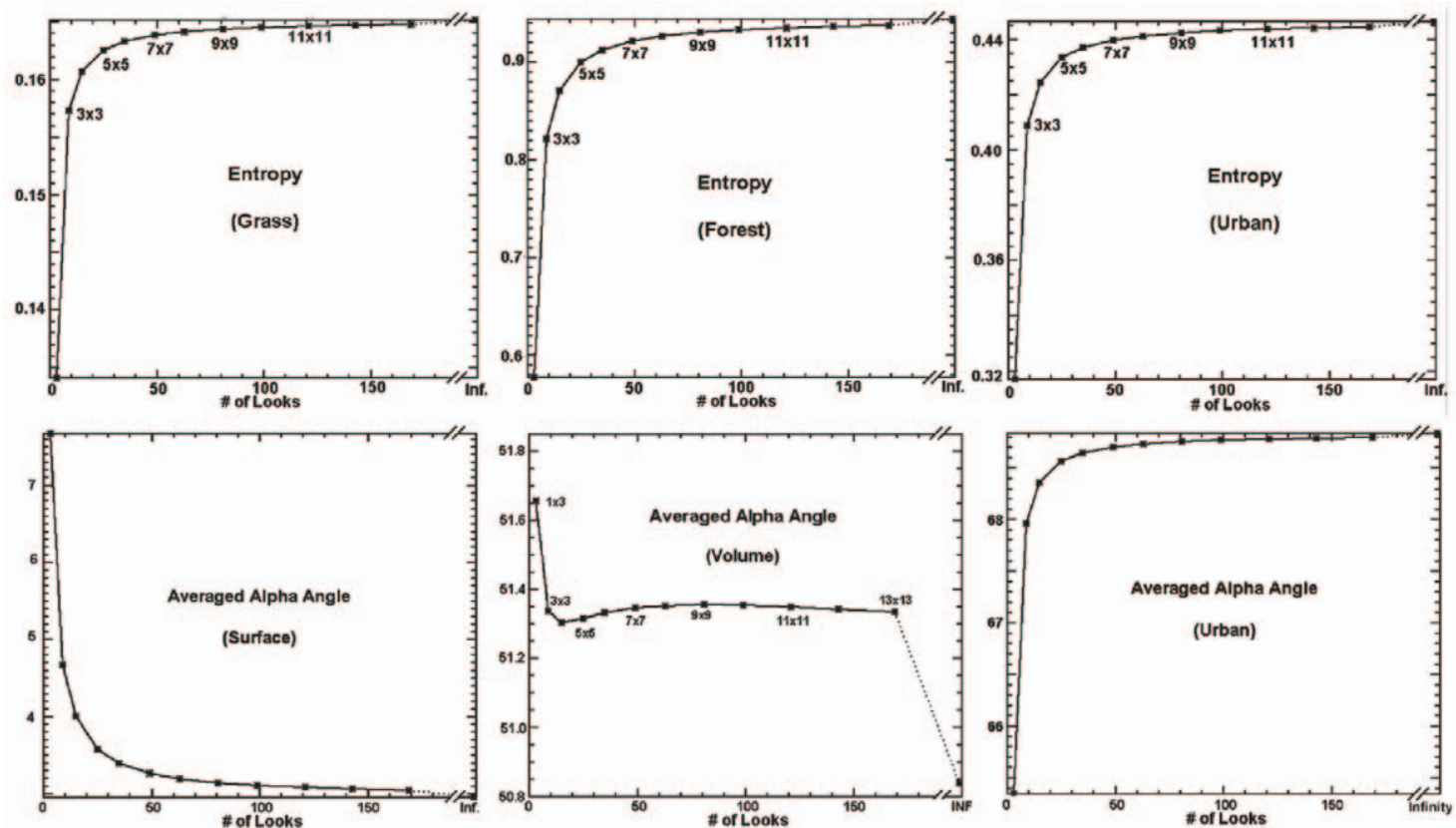

Fig. 3. Behaviors of the Entropy and Averaged alpha angle with various numbers of looks for grass areas, forested areas, and urban (Lee et al., 2008). 


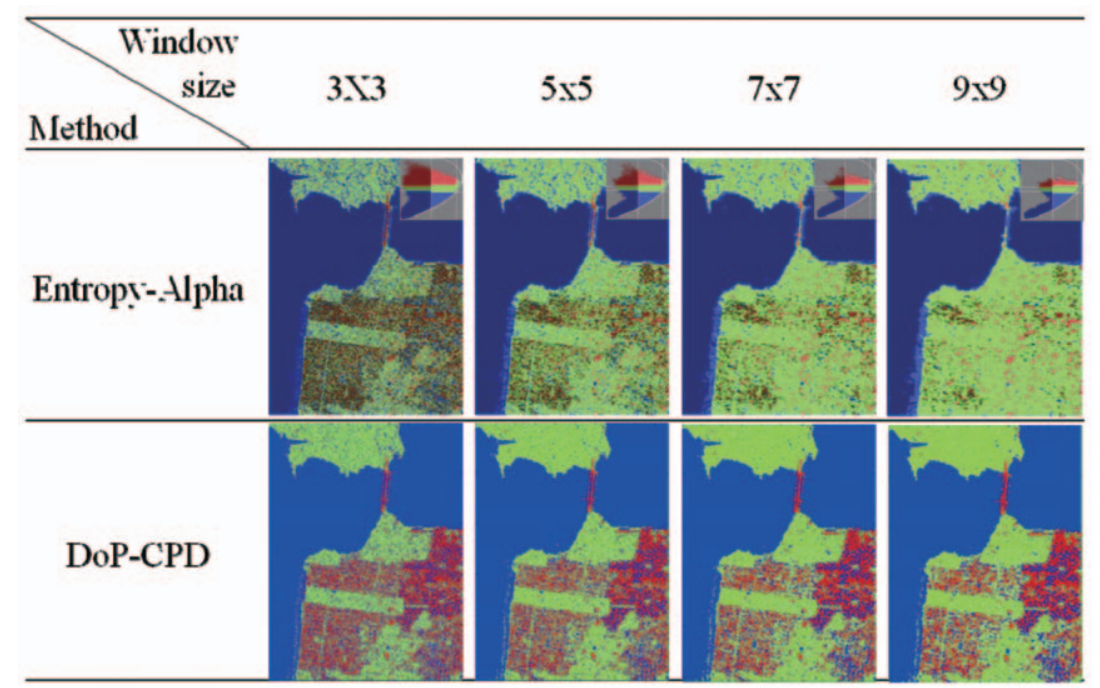

Fig. 4. Comparison of classification results with various window sizes for the Entropy-Alpha technique and the DoP-CPD technique.

number of imaging pixels for averaging. We started from $3 \times 3$ pixels for the ensemble average and computed the parameters with $5 \times 5,7 \times 7$, and $9 \times 9$ pixels to evaluate the effect of ensemble average on these parameters. The DoP decreases as the number of averaging pixels increases for short vegetation and tall vegetation areas, while the DoP does not show noticeable change for bare surface and urban areas with increasing the number of averaging pixels, as shown in Fig. 1. The CPD also decreases with an increase of the number of averaging pixels for short vegetation and tall vegetation areas, and almost insensitive on the number of averaging for bare surface and urban areas, as shown in Fig. 2.

Recently, Lee et al. (2008) analyzed the behavior of sample average on $H, A$, and $\bar{\alpha}$. In general, entropy will be underestimated and anisotropy will be overestimated, if the number of looks is insufficiently large. They also found that the bias in alpha angle can be either under or overestimated depending on scattering mechanism. Fig. 3 shows Behaviors of the Entropy and Averaged alpha angle with various numbers of looks (Lee et al., 2008). Fig. 4 shows a comparison between the entropy-alpha technique and the DoP-CPD technique on polarimetric SAR image classification with various numbers of looks.

It is shown in Fig. 4 that the DoP-CPD is less sensitive to the number of averaging pixels than the entropy-alpha technique.

\section{DoP-CPD diagram}

The SAR image pixels of a bare surface and a building area have high DoP values because the single scattering and double bounce scattering are dominant in the areas. On the other hand, the pixels of a forest and a vegetation area have low DoP values because of the multiple scattering effects in the areas. The CPD values of SAR image pixels are also dependent of the various scattering mechanisms: single bounce scattering, double bounce scattering and volume scattering.

The DoP and CPD values of each pixel of a SAR image are positioned in the DoP-CPD diagram with the vertical axis of the DoP and the horizontal axis of the CPD. We first analyzed the DoP and the CPD distributions over the DoP-CPD diagram for various 


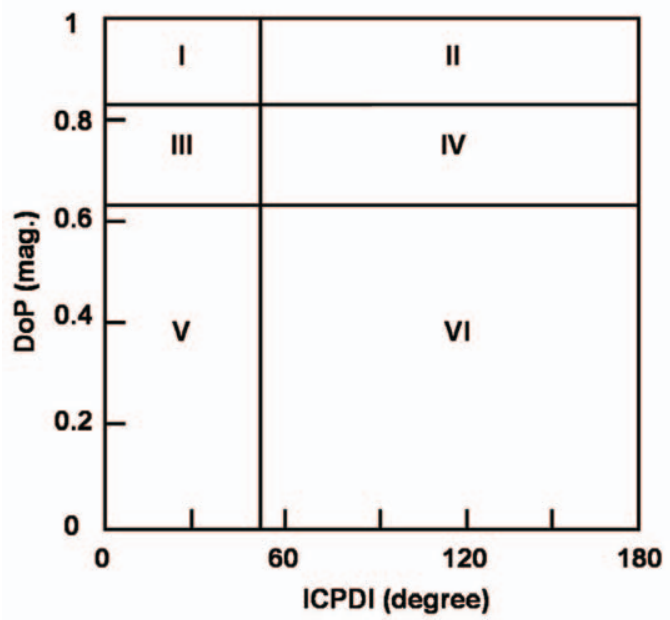

Fig. 5. The DoP-CPD diagram for classification of polarimetric SAR images.

ground types; for example, a bare surface, a village area, a short vegetation area, and a forest area. We then set empirically the boundaries of the DoP and the CPD between the classes based on the DoP-CPD distributions of various target areas. We analyzed the sensitivities of the classification parameters on various targets and the effect of ensemble averaging. Then, the optimum values of the DoP for class separations have been chosen to be 0.85 and 0.65 according to the degree of multiple scattering, as shown in Fig. 5. The CPD has been used to separate the dominant scattering mechanisms between singleand double-bounce scatterings. Based on the data analysis, we empirically chose the optimum boundary value with $45^{\circ}$, as shown in Fig. 5.

The existing diagram was used from $-180^{\circ}$ to $180^{\circ}$ of CPD. In this paper we propose a diagram using absolute $\mathrm{CPD}$. Because, based on $0^{\circ}, \mathrm{CPD}$ are similar to value of both side.

Each class of the DoP-CPD diagram (Fig. 5) can be summarized as follows:

- Zone I (DoP>0.85, ICPD $\left.<45^{\circ}\right)$ : Single-bounce surface scattering areas such as bare soil surfaces and water surfaces.
- Zone II (DoP>0.85, $\left.|\mathrm{CPD}|>45^{\circ}\right)$ : Double-bounce surface scattering areas such as a town or village with buildings.

- Zone III $\left(0.65<\mathrm{DoP}<0.85, \mathrm{ICPD}<45^{0}\right)$ : Singlebounce surface scattering is mixed with volume scattering, as in short-vegetated farming fields.

- Zone IV $\left(0.65<\mathrm{DoP}<0.85,|\mathrm{CPD}|>45^{0}\right)$ : Doublebounce surface backscatters beneath a sparse crown layer having randomly oriented leaves and branches.

- Zone V (DoP<0.65, $\left.|\mathrm{CPD}|<45^{0}\right)$ : Volume scattering areas such as deciduous forests or a dense short vegetation fields.

- Zone VI $\left(\mathrm{DoP}<0.65,|\mathrm{CPD}|>45^{0}\right)$ : Doublebounce backscattering from a trunk layer with random scattering through a sparse crown layer, as in sparse coniferous forests.

\section{Verification of the DoP-CPD classification technique with RADARSAT-2}

The DoP-CPD diagrams are verified with the selected study areas (San Francisco) at first. Fig. 6 shows a DoP-CPD diagram with the pixel distributions of the DoP and CPD for a RADARSAT-2 image for

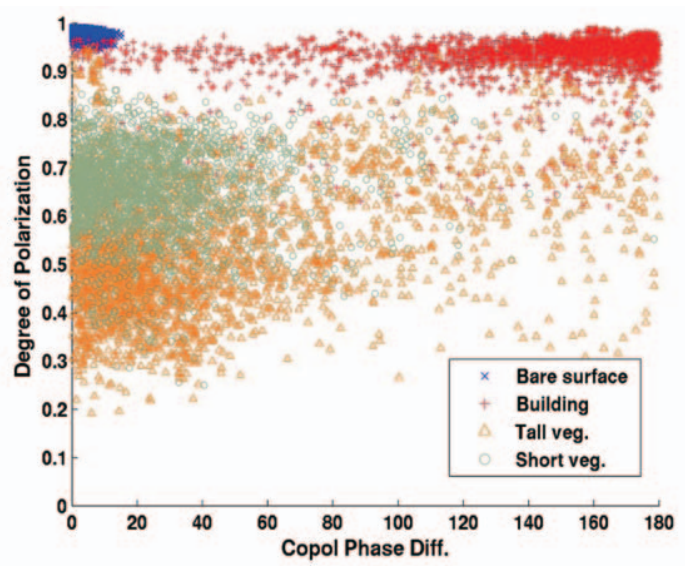

Fig.6. Classification results of a RADARSAT-2 image. 
Table 1. Accuracy of the classification results

\begin{tabular}{c|c|c|c|c|c|c|c|c}
\hline \hline \multirow{2}{*}{ Zone } & \multicolumn{2}{|c|}{ Bare surface } & \multicolumn{2}{c|}{ Village } & \multicolumn{2}{c|}{ Short veg. } & \multicolumn{2}{c}{ Tall veg. } \\
\cline { 2 - 9 } & $\mathrm{N}$ & $\%$ & $\mathrm{~N}$ & $\%$ & $\mathrm{~N}$ & $\%$ & $\mathrm{~N}$ & $\%$ \\
\hline I & 2500 & 100 & 67 & 2.68 & 3 & 0.12 & 15 & 0.6 \\
\hline II & 0 & 0 & 2276 & 91.04 & 2 & 0.08 & 8 & 0.32 \\
\hline III & 0 & 0 & 29 & 1.16 & 1544 & 61.76 & 154 & 6.16 \\
\hline IV & 0 & 0 & 114 & 4.56 & 76 & 3.04 & 89 & 3.56 \\
\hline V & 0 & 0 & 6 & 0.24 & 827 & 33.08 & 1980 & 79.2 \\
\hline VI & 0 & 0 & 8 & 0.32 & 48 & 1.92 & 254 & 10.16 \\
\hline Total & 2500 & 100 & 2500 & 100 & 2500 & 100 & 2500 & 100 \\
\hline \hline
\end{tabular}
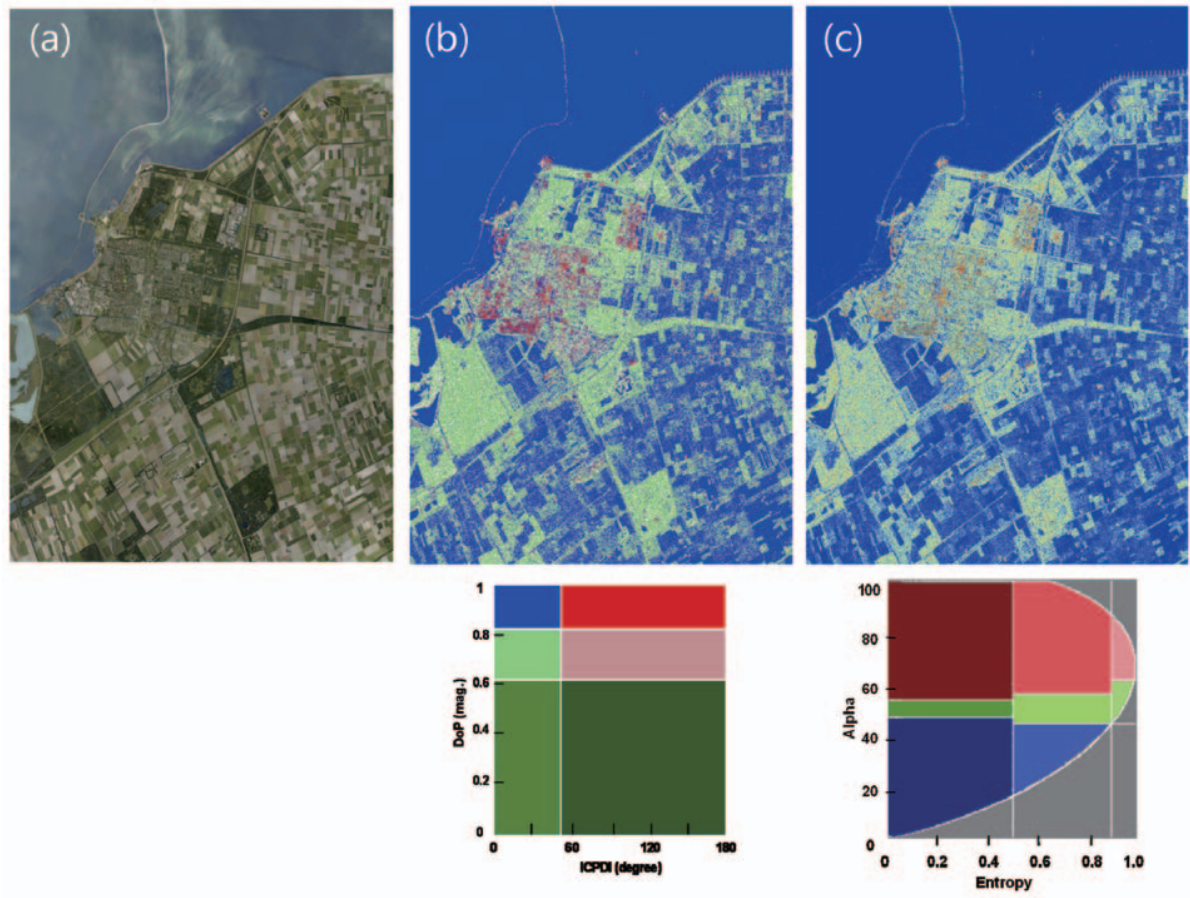

Fig. 7. Comparison of classification techniques with a polarimetric RADARSAT-2 image: (a) Aerial photo, (b) DoP-CPD classification, (c) Entropy- alpha classification.

the bare surfaces $(\mathrm{x})$, village areas $(+)$, short vegetation areas (o), and tall vegetation areas $(\Delta)$.

Table 1 shows the corresponding classification results using the DoP-CPD classification technique. Surprisingly, all pixels (2500 pixels) of the bare surface are classified to the 'Zone I' of the DoP-CPD diagram. Most pixels (91.04\%) of the town (village area) are classified to the 'Zone II' of the DoP-CPD diagram. For the short vegetation fields, only $61.76 \%$ of the pixels are classified to Zone III. For the tall vegetation fields, $79.2 \%$ of the pixels are classified to Zone V. About $10 \%$ of the pixels are classified to Zone VI, because the double bounce scattering may occur for a sparse forest area.

For more verification, DoP-CPD classification technique has been applied to RADARSAT-2 images taken over the Flevoland, Netherlands. Fig. 7 shows qualitatively the classification results of a polarimetric RADARSAT-2 image taken over the Flevoland area of the DoP-CPD technique, which was compared 
with the entropy-alpha classification techniques. Flevoland is most of crop land, there are some of urban. In general, for an urban area, although backscattered signals in general are from singlebounce, double-bounce and multiple-bounce returns of various parts of the structure, the double-bounce scattering is still dominant for urban areas. So, double bounce objects exhibiting 'red' in general classification scheme. The DoP-CPD can see more 'red' color than the entropy-alpha technique in urban areas as shown in Fig. 7.

\section{Concluding remarks}

Based on the analyses for the DoP and CPD for various earth terrains, a simple unsupervised classification technique for polarimetric SAR images has been evaluated. We then set empirically the boundaries of the DoP and the CPD between the classes based on the DoP-CPD distributions of various target areas. We analyzed the sensitivities of the classification parameters on various targets and the effect of ensemble averaging. The DoP-CPD is less sensitive to the number of averaging pixels than the entropy-alpha technique. It would be a stronger statement and better proof. The accuracy of the simple classification technique was examined with Cband polarimetric RADARSAT-2 data. Although this classification technique using the DoP and CPD is simple, the DoP-CPD diagram might be a good tool for classifying polarimetric SAR images

\section{Acknowledgment}

The authors would like to thank the anonymous reviewers for their thoughtful comments. The authors also thank MDA for their help in the polarimetric SAR data acquisition.

\section{References}

Cloude, S.R. and E. Pottier, 1997. An Entropy based classification scheme for land applications of polarimetric SAR, IEEE Transactions on Geoscience and Remote Sensing, 35(1): 68-78.

Elies, P., B.L. Jeune, P. Olivard, J. Cariou, and J. Lotrian, 1997. The application of depolarization analysis to polarimetric characterization and classification of metallic and dielectric samples, Journal of Physics D: Applied Physics, 30: 2520-2529.

Ersahin, K., I.G. Cumming, and R.K. Ward, 2010. Segmentation and Classification of Polarimetric SAR Data Using Spectral Graph Partitioning, IEEE Transactions on Geoscience and Remote Sensing, 48(1): 164-174.

Freeman, A. and S.L. Durden, 1998. A threecomponent scattering model for polarimetric SAR data, IEEE Transactions on Geoscience and Remote Sensing, 36(3): 963-973.

Kong, J.A., A.A. Swartz, H.A. Yueh, L.M. Novak, and R.T. Shin, 1988. Identification of terrain cover using the optimum polarimetric classifier, Journal of Electromagnetic Waves Applications, 2: 171-194.

Lardeux, C., P.L. Frison, C. Tison, J.C. Souyris, B. Stoll, B. Fruneau, and J.-P. Rudant, 2009. Support Vector Machines for Multifrequency SAR Polarimetric Data Classification, IEEE Transactions on Geoscience and Remote Sensing, 47(12): 4143-4142.

Lee, J.S., M.R. Grunes, and R. Kwok, 1994 Classification of multilook polarimetric SAR imagery based on complex Wishart distribution, International Journal of Remote Sensing, 15(11): 2299-2311.

Lee, J.S. and E. Pottier, 2009. Polarimetric Radar 
Imaging from Basics to Application, CRC press, pp. 48-49.

Lee, J.S., T.L. Ainsworth, J.P. Kelly, and C. LopezMartinez, 2008. Evaluation and Bias Removal of Multilook Effect on Entropy/ Alpha/Anisotropy in Polarimetric SAR Decomposition, IGARSS 2007 Special issue, IEEE Transactions on Geoscience and Remote Sensing, 46(10): 3039-3052.

Lee, K.Y. and Y. Oh, 2010. New simple decomposition technique for polarimetric SAR images, Korean Journal of Remote Sensing, 26(1): 1-7.

Oh, Y., K.Y. Lee, and G. Jang, 2009. New unsupervised classification technique for polarimetric SAR images, Korean Journal of Remote Sensing, 25(3): 255-261.

Rio, V.S., J.M. Mosquera, V. Isasa, and M.E. Lorenzo, 2006. Statistics of the degree of polarization, IEEE Transactions on Antennas and Propagation, 54(7): 2173-2175.
Sinclair, G., 1950. The transmission and reception of elliptically polarized waves, Proc. IRE, 38(2): 148-151.

Ulaby, F.T. and C. Elachi, 1990. Radar Polarimetry for Geoscience Applications. Artech House Remote Sensing Library, pp. 4-16.

Ulaby, F.T., K. Sarabandi, and A. Nashashibi, 1992. Statistical properties of the Mueller matrix of distributed targets, IEE Proc-F, 139(2): 136-146.

Van zyl, J.J., C.H. Papas, and C. Elachi, 1987. On the optimum polarization of incoherently reflected waves, IEEE Transactions on Antennas and Propagation, 35(7): 818-825.

Yamaguchi, Y., T. Moriyama, M. Ishido, and H. Yamada, 2005. Four-component scattering model for polarimetric SAR image decomposition, IEEE Transactions on Geoscience and Remote Sensing, 43(8): 16991706. 